\title{
Short and long-term sensitivity of lab-scale thermocline based thermal storage to flow disturbances
}

\author{
Sandeep Hatte ${ }^{\mathrm{a}}$, Carolina Mira-Hernández ${ }^{\mathrm{b}}$, Advaith S. ${ }^{\mathrm{a}, \mathrm{c}}$, Aashay Tinaikar ${ }^{\mathrm{a}}$, Utpal Kumar Chetia ${ }^{\mathrm{a}}$, \\ Manu K. V. ${ }^{\text {a }}$, Kamanio Chattopadhyay ${ }^{\mathrm{c}}$, Justin A. Weibel ${ }^{\mathrm{b}}$, Suresh V. Garimella ${ }^{\mathrm{b}}$, Vinod \\ Srinivasan $^{\mathrm{d}}$ and Saptarshi Basu ${ }^{\mathrm{a}, \mathrm{c}, *}$ \\ ${ }^{a}$ Department of Mechanical Engineering, Indian Institute of Science, Bangalore, 560012, India \\ ${ }^{\mathrm{b}}$ School of Mechanical Engineering, Purdue University, West Lafayette, IN 47907-2088, USA \\ ${ }^{\mathrm{c}}$ Interdisciplinary Centre for Energy Research (ICER), Indian Institute of Science, Bangalore, \\ 560012, India. \\ ${ }^{d}$ Department of Mechanical Engineering, University of Minnesota, Minneapolis, MN 55455, USA \\ *Corresponding Author email: sbasu@mecheng.iisc.ernet.in
}

\begin{abstract}
Molten-salt thermocline-based systems are a low-cost option for single-tank thermal energy storage in concentrated solar power plants. Due to the high variability in solar energy availability, these energy storage devices are subject to transient heat loads during charging that can affect the storage efficiency. Numerical simulations were conducted to analyze the stability characteristics of a labscale thermocline tank subject to a flow disturbance during charging under different operating temperatures. The charging process was first simulated at a constant Reynolds number for three different Atwood numbers; a stably stratified fluid layer develops inside the storage tank in all cases. A flow disturbance was then introduced at the inlet of the stratified thermocline tank by inserting colder fluid for a short period of time. The disturbance interacts with the thermocline and causes oscillations and mixing. The thermocline oscillations are under-damped and lead to an increase in thermocline region thickness. The transient behavior of the thermocline and the decay rate in its oscillations were analyzed; the damping time depends on the Atwood number. The persistence of flow disturbance effects during long-term cyclical operation was also investigated. Several charge/discharge cycles were simulated at constant Reynolds number to obtain a timeperiodic thermal response for each Atwood number. The characteristic flow disturbance was introduced at the inlet during a single charging process, and the thermocline region was observed during several subsequent charge/discharge cycles to assess the long-term temporal attenuation of the disturbance. The thermocline almost fully recovers to the time-periodic behavior after a single cycle.
\end{abstract}

Keywords: Solar thermal energy storage, thermocline, Rayleigh-Taylor instability, thermal storage efficiency 


\section{Nomenclature}

At Atwood number $\left(\left(\rho_{c}-\rho_{h}\right) /\left(\rho_{c}+\rho_{h}\right)\right)$, -

$\mathrm{At}_{d} \quad$ Disturbance Atwood number $\left(\left(\rho_{d}-\rho_{h}\right) /\left(\rho_{d}+\rho_{h}\right)\right)$

$c_{p} \quad$ Specific heat, $\mathrm{kJ} / \mathrm{kgK}$

$D \quad$ Diameter of the tank, $\mathrm{m}$

E Specific energy, $\mathrm{J} / \mathrm{kg}$

$g \quad$ Acceleration due to gravity, $\mathrm{m} / \mathrm{s}^{2}$

$H \quad$ Height of the tank, $\mathrm{m}$

$h^{*} \quad$ Non-dimensional axial distance $(y / H)$, -

$k \quad$ Thermal conductivity, $\mathrm{W} / \mathrm{mK}$

$L_{P} \quad$ Penetration length, $\mathrm{m}$

$L_{p}{ }^{*} \quad$ Non-dimensional penetration length, $\left(L_{P} / H\right)$, -

$\dot{m} \quad$ Mass flow rate, $\mathrm{kg} / \mathrm{s}$

$N \quad$ Brunt-Väisälä frequency $\left((-g / \rho(\mathrm{d} \rho / \mathrm{d} y))^{1 / 2}\right), \mathrm{Hz}$

$p \quad$ Pressure, $\mathrm{Pa}$

$r \quad$ Radial direction, $\mathrm{m}$

$r^{*} \quad$ Non-dimensional radial distance $(2 r / D)$, -

Re Reynolds number $\left(\rho_{h} v_{c} D / \mu_{h}\right)$

Ri Richardson number $\left((-g \nabla \rho) /\left(\rho(\nabla v)^{2}\right)\right)$, -

$T \quad$ Temperature, ${ }^{\circ} \mathrm{C}$

$\bar{T} \quad$ Mass-weighted average temperature, ${ }^{\circ} \mathrm{C}$

$t \quad$ Time, $\mathrm{s}$

$T^{*} \quad$ Non-dimensional temperature $\left(\left(T-T_{c}\right) /\left(T_{h}-T_{c}\right)\right)$, -

$t_{0} \quad$ Half-cycle period, $\mathrm{s}$

$T_{c} \quad$ Cold fluid temperature, ${ }^{\circ} \mathrm{C}$

$T_{d} \quad$ Disturbance fluid temperature, ${ }^{\circ} \mathrm{C}$

$T_{h} \quad$ Hot fluid temperature, ${ }^{\circ} \mathrm{C}$

$T_{j}^{*} \quad$ Non-dimensional temperature in $\mathrm{j}^{\text {th }}$ cycle, -

$T_{m}{ }^{*} \quad$ Non-dimensional mean temperature inside the thermocline region, -

$u \quad$ Radial velocity, $\mathrm{m} / \mathrm{s}$

$u^{*} \quad$ Non-dimensional radial velocity $\left(u / v_{c}\right)$, -

$v \quad$ Axial velocity, $\mathrm{m} / \mathrm{s}$

$v^{*} \quad$ Non-dimensional axial velocity $\left(v / v_{c}\right)$, -

$v_{c} \quad$ Characteristic inlet velocity, $\mathrm{m} / \mathrm{s}$ 
$v_{d} \quad$ Inlet velocity during flow disturbance, $\mathrm{m} / \mathrm{s}$

$y \quad$ Axial (vertical) direction, $\mathrm{m}$

Greek

$\delta \quad$ Thermocline thickness, $\mathrm{m}$

$\delta^{*} \quad$ Non-dimensional thermocline thickness $(\delta / H)$, -

$\eta_{\mathrm{I}} \quad$ First law efficiency,-

$\mu \quad$ Viscosity, $\mathrm{Ns} / \mathrm{m}^{2}$

$\rho \quad$ Density, $\mathrm{kg} / \mathrm{m}^{3}$

$\rho_{c} \quad$ Fluid density at cold fluid temperature, $\mathrm{kg} / \mathrm{m}^{3}$

$\rho_{d} \quad$ Fluid density at disturbance fluid temperature, $\mathrm{kg} / \mathrm{m}^{3}$

$\rho_{h} \quad$ Fluid density at hot fluid temperature, $\mathrm{kg} / \mathrm{m}^{3}$

$\tilde{\tau} \quad$ Viscous stress tensor, $\mathrm{Pa}$

$\tau_{\text {in }} \quad$ Non-dimensional time for initial charging process $\left(t v_{\mathrm{c}} / H\right)$, -

$\tau_{c} \quad$ Time scale for Rayleigh-Taylor instability $\left(\left(H /\left(\mathrm{At}_{d} g\right)\right)^{1 / 2}\right), \mathrm{s}$

$\tau^{*} \quad$ Non-dimensional time during disturbance evolution $\left(t / \tau_{c}\right)$, -

$\tau_{\text {damp }}^{*}$ Non-dimensional damping time constant, -

$\tau_{\delta o}^{*} \quad$ Non-dimensional time of inception of deformation, -

$\tau_{m o}^{*} \quad$ Non-dimensional time of inception of mixing, -

$\tau_{\operatorname{lag}}^{*} \quad$ Non-dimensional time lag between inception of deformation and mixing $\left(\tau_{m o}^{*}-\tau_{\delta o}^{*}\right),-$

\section{Introduction}

Anthropogenic climate change calls for urgent international action to reduce the consumption of fossil fuels while simultaneously increasing the accessibility of reliable power sources to developing nations; such action has focused research and development efforts on cost-effective and clean-energy technologies. Concentrated solar power (CSP) systems are one promising technology for large-scale conversion of solar energy to electricity. In CSP plants, mirrors or lenses are used to concentrate solar irradiation and heat a working fluid that can be used to ultimately generate electricity; when thermal energy storage systems are used in conjunction with CSP systems, complications associated with high variability of solar energy can be mitigated. With further technological development, it has been estimated that CSP systems could satisfy $9.6 \%$ of global electricity demand by the year 2050 [1]. Low-cost energy storage that provides a continuous source of power during periods of low sunlight remains one of the major challenges in widespread deployment of CSP systems. 
In CSP plants, a thermal energy storage (TES) system can be used to address the mismatch between the demand and supply of energy [2]. TES systems can employ molten salt, commonly a eutectic mixture of sodium and potassium nitrate, as the heat transfer fluid (HTF) for hightemperature CSP applications. Molten salt has an advantage over other candidate fluids such as organic oils due to a wider working temperature range, better thermal stability and low vapour pressure [3]. Sensible TES using molten salts can be either two-tank or single-tank thermocline storage. In a two-tank storage system, hot- and cold-temperature molten salt inventories are stored in separate tanks. In a single-tank thermocline storage system, hot and cold molten salt reserves are stored in an inherently stable stratified configuration, eliminating the cost of one of the tanks [4]. Additional cost reduction can be achieved by introducing a low-cost filler material inside the single tank to provide thermal capacity while displacing molten salt volume. Energy storage in a single thermocline tank with filler material reduces the cost by $33 \%$ as compared to a two-tank system [5]. As such, a thermocline tank TES system is economically promising for large-scale storage and distribution of solar power [6].

Operation of a thermocline tank thermal energy storage system involves charging and discharging processes. During charging, hot fluid coming from the solar collection field enters at the top of the tank as cold fluid exits from the bottom to be heated. During discharging, hot fluid from the top is pumped to the power block to generate electricity and returns to the tank at the bottom at a relatively colder temperature. Heat exchange between hot and cold portions of the fluid results in a region experiencing a high temperature gradient known as 'thermocline'. The stratification efficiency of the tank can be quantified by measuring the thickness of this thermocline region. A tank with a thin thermocline exhibits superior storage efficiency compared to a tank with a thicker thermocline. The thickness of the thermocline depends on various factors like the temperature difference between hot and cold fluid, fluid properties and charging rate $[4,7,8]$.

Thermal stratification inside a thermocline tank can be adversely affected by various flow instabilities that can cause undesirable mixing of hot and cold portions of the fluid, which thickens the thermocline region. In thermocline tanks with solid filler material, viscous fingering and channeling can develop from small disturbances under unstable flow conditions [7]. Diurnal variation in solar radiation also makes it difficult to maintain a constant hot fluid inlet temperature during charging. Relatively colder (denser) fluid may enter the tank and disturb the stable thermocline, thereby increasing its thickness [9]. Rayleigh-Taylor instabilities are observed under such circumstances inside thermocline tanks without filler material. Numerical simulations performed by Manu et al. [10,11] have shown that Rayleigh-Taylor instabilities arising from random perturbations can cause temporary break-down of the thermocline region. The disturbances initially grow exponentially, followed by non-linear stages during formation of mushroom-like and 
falling spikes-like structures. These evolved perturbations can interact with the thermocline region causing de-stratification [10].

The efficiency of a thermocline tank disturbed by Rayleigh-Taylor instabilities is governed by the entrainment of cold fluid into the hot fluid. De-stratification caused by the spike-like structures augments mixing across the interface. The impact of flow structures on a sharp density interface has been analyzed in previous studies [12]. For a sharp interface, the subsequent mixing dynamics is essentially governed by the ratio of buoyancy and inertia forces described by the Richardson number (Ri) [13]. A low value of Richardson number $(\mathrm{Ri}<1)$ indicates a low degree of stratification. In such cases, perturbations can effortlessly penetrate the thermocline region. With an increase in the Richardson number, the thermal stratification is more resilient to perturbations.

Impingement of fluid structures on the thermocline leads to a variety of internal wave motions inside the tank. The variation in density along the direction of stratification resists the vertical motion of fluid. In such cases, the observed oscillation frequencies in the thermocline region are normally less than the Brunt Väisälä frequency $(N)$ [14]. Y. Noh et al. [15] investigated the impact of thermal disturbances on a sharp density interface and studied the waves generated at the interface. They found that the inertia of the incoming fluid is the primary source of disturbance, which causes oscillations in the thermocline. Kaloudis [16] numerically simulated the flow phenomena during the discharging of a thermally stratified water tank for energy storage. The vertical velocity monitored at different positions showed oscillations occurring in the thermocline region.

Thermocline TES systems are mainly designed using empirical correlations. The limited existing measurements of temperature and velocity in thermocline tanks are of low resolution and provide little insight into the nature of instabilities that may occur. Computational fluid dynamics simulations yield highly resolved temperature and velocity fields that can be used for assessing the impact of instabilities inside the thermocline tank.

Previous numerical studies of Rayleigh-Taylor instabilities in thermocline tanks were focused on analyzing the effect of Reynolds number and inflow temperature on thermal stratification [10]. However, these studies did not quantify the spatio-temporal dynamics of the thermocline during and after vortical interactions; furthermore, the impact of the disturbances on the overall storage efficiency has not been investigated. In the present work, the sensitivity of a thermocline tank to a flow disturbance is studied for different Atwood numbers (non-dimensional density difference). A short-term half charge disturbance cycle analysis studies the interaction of the thermocline region with a flow disturbance induced at the top of an initially stratified tank. A long-term multiple cycle analysis then quantifies the effects of this flow disturbance on the thermal performance of the tank under cyclical operation. 
The parameters used in the simulation are chosen to match a lab-scale thermocline tank system in the Interdisciplinary Centre for Energy Research (ICER) at IISc Bangalore. This tank has a diameter of $0.15 \mathrm{~m}$ and a height of $1.00 \mathrm{~m}$ and uses a commercial eutectic mixture (known as solar salt) composed of sodium nitrate $(60 \mathrm{wt} \%)$ and potassium nitrate $(40 \mathrm{wt} \%)$ that solidifies at $221{ }^{\circ} \mathrm{C}$ as the heat transfer fluid.

\section{Numerical Model}

Short- and long-term analyses of the thermocline tank sensitivity to a flow disturbance during charging are conducted. Schematic diagrams of the computational domain and boundary conditions for the different analyses are shown in Figure 1.

\subsection{Problem description}

The thermocline tank hot source temperature $\left(T_{h}\right)$ is fixed at $350{ }^{\circ} \mathrm{C}$, while three different cold source temperature $\left(T_{c}\right)$ values of $340{ }^{\circ} \mathrm{C}, 300{ }^{\circ} \mathrm{C}$ and $250{ }^{\circ} \mathrm{C}$, corresponding to Atwood numbers (At) of $0.00167,0.0084$ and 0.0167, are evaluated to analyze the effect of Atwood number on thermal stratification. Charging and discharging processes occur at a constant flow rate of $81 / \mathrm{min}$, which corresponds to a Reynolds number (Re) of 905 , with a characteristic charging velocity $\left(v_{c}\right)$ of $7.55 \times 10^{-3} \mathrm{~m} / \mathrm{s}$. The following polynomial curve fits derived from experimental data are used for defining the temperature-dependent physical properties of molten salt (temperature in Celsius) [3].

$$
\begin{gathered}
\rho(T)=2090-0.636 T \\
c_{p}(T)=1443-0.172 T \\
k(T)=0.443+1.9 \times 10^{-4} T \\
\mu(T)=22.714 \times 10^{-3}-0.120 \times 10^{-3} T+2.281 \times 10^{-7} T^{2}-1.474 \times 10^{-10} T^{3}
\end{gathered}
$$

\subsubsection{Short-term sensitivity to a flow disturbance}

This analysis investigates the phenomena that occur after a pulse of fluid, with temperature lower than that of the hot source, is introduced at the top of the initially stratified thermocline tank. The growth of the disturbance, its interaction with the thermocline and the resultant changes inside the tank are studied. The initial stratified condition is obtained by simulating a charging process with hot source fluid at constant flow rate of an initially isothermal tank (Figure 1a) until the thermocline reaches half the tank height $(t=66.2 \mathrm{~s})$. After the charging process, an additional period (duration of $134 \mathrm{~s}$ ) without inflow is simulated that allows buoyancy forces to remove radial temperature gradients, which form due to frictional resistance at the wall during charging. The flow disturbance $\left(T_{d}=345{ }^{\circ} \mathrm{C}\right.$ ) is introduced at the top of the tank for $25 \mathrm{~s}$ with a parabolic velocity profile (Figure 
1b). An additional period without inflow is simulated after the disturbance for a duration of $775 \mathrm{~s}$. The interaction of the disturbance with the thermocline and consequent thickening of the thermocline region are studied. All the results in this work pertain to the chosen, lab-scale tank geometry described above.

\subsubsection{Long-term sensitivity to a flow disturbance}

This analysis investigates persistence of a single flow disturbance under cyclic chargingdischarging operation. First, several charging and discharging processes with uniform and constant inflow of fluid at the hot and cold temperatures, respectively, are simulated until a time-periodic response is obtained (Figure 1c). Then, a charging process is simulated in which the same disturbance as in the short-term study is applied. Subsequently, another series of cyclic chargingdischarging operations are simulated to assess the attenuation of the disturbance effects in the longer term (Figure 1d). The reestablishment of thermal stratification inside the tank and deterioration in thermal performance after the flow disturbance are evaluated.

During cyclical operation, the charging and discharging processes are of equal duration, $t_{0}=120 \mathrm{~s}$. Hence, the thermocline tank is never fully charged or discharged, and a fraction of the thermocline region is always retained inside the tank. In power plants, thermocline tanks are operated such that a portion of the thermocline region always remains inside the tank to prevent sending overly hot fluid to the solar collection field or cold fluid to the power block $[17,18]$. After a time-periodic response is obtained, a charging process is simulated that includes a flow disturbance. This charging process consists of two stages of normal charging with hot fluid each lasting $47.5 \mathrm{~s}$, with inflow of the disturbance lasting $25 \mathrm{~s}$ in between these two stages. After the chargingdischarging cycle with the flow disturbance, 10 additional charging-discharging cycles are simulated to assess the persistence of the disturbance effects.

\subsection{Governing equations and boundary conditions}

Thermal phenomena inside the thermocline tank are governed by conservation of mass, momentum, and energy, which are respectively:

$$
\begin{gathered}
\frac{\partial \rho}{\partial t}+\nabla \cdot(\rho \vec{v})=0 \\
\frac{\partial}{\partial t}(\rho \vec{v})+\nabla \cdot(\rho \vec{v} \vec{v})=-\nabla p+\nabla \cdot \tilde{\tau}+\rho \vec{g} \\
\frac{\partial}{\partial t}(\rho E)+\nabla \cdot(\vec{v}(\rho E+p))=\nabla \cdot(k \nabla T)
\end{gathered}
$$


To isolate and study the effects of well controlled disturbances on the response and degradation of the thermocline, laminar flow conditions (low Reynolds Number) are used and viscous dissipation effects are neglected. Fluid flow is assumed to be axisymmetric. The preliminary experimental observations for the parameter space investigated here show that the evolutions of disturbances are mostly axisymmetric. In that context, 2D simulation allows a good approximation of the thermocline degradation mechanisms.

During normal charging processes, the boundary condition at the top of the domain is set as a uniform velocity inlet $\left(v_{c}\right)$ and temperature $\left(T_{h}\right)$; the boundary condition at the bottom is set as a pressure outlet. During normal discharging processes, the boundary condition at the top of the domain is set as a pressure outlet, while the boundary condition at the bottom is set as a uniform velocity inlet $\left(v_{c}\right)$ and temperature $\left(T_{\mathrm{c}}\right)$. During periods of zero inflow, the top and the bottom of the tank are modeled as adiabatic no-slip walls. During an induced flow disturbance, the boundary condition at the top is set as a velocity inlet with parabolic profile:

$$
v_{d}(r)=2 v_{c}\left[1-\left(\frac{2 r}{D}\right)^{2}\right]
$$

The boundary condition at the bottom is set as a pressure outlet. For the entire duration of the simulations, the side walls are considered to be adiabatic with a no-slip boundary condition. Parameters like wall heat loss and secondary effects like natural convection have been considered negligible, so as to assess the degradation of thermal stratification and the thermocline response due to flow disturbances only.

For the short-term analysis, the initial condition is an isothermal cold tank with zero velocity. For the long-term analysis, in order to expedite the convergence to a time-periodic solution, an initial temperature distribution is imposed. The temperature in the upper half of the thermocline tank is set to the hot fluid temperature, and the temperature in the lower half is set to the cold fluid temperature. The initial velocity is set to zero. The solution is considered timeperiodic converged when the non-dimensional temperature difference between instants corresponding to the end of the charging process in consecutive cycles $\left(T_{j}^{*}-T_{j-1}^{*}\right)$ drops below $10^{-5}$. The thermocline tank achieves a time-periodic response after 12 charging-discharging cycles.

\subsection{Solution procedure}

The governing equations were numerically solved using the finite volume method implemented in the commercial computational fluid dynamics software FLUENT [19]. The solution domain was 
discretized into rectangular cells, with 500 divisions along the height and 150 along the radius. The grid-independence was assessed by comparing the simulation results for a single case during evolution of the flow disturbance (At $=0.00844$ at $t=10 \mathrm{~s}$ ) for grids with $250 \times 75$ and $1000 \times 300$ elements; the maximum velocity difference was within $0.34 \%$ and the temperature within $0.08 \%$. The pressure implicit with splitting of operators (PISO) algorithm was used for pressure-velocity coupling [20]. A second-order upwind discretization scheme was used for the energy and momentum equations, while a body force-weighted scheme was used for pressure terms. Underrelaxation factors for pressure and momentum were fixed at 0.3 and 0.7 , respectively. A convergence criterion of $10^{-4}$ was used for residuals of the continuity and momentum equations; the criterion was $10^{-10}$ for the energy equation. A time step of $0.1 \mathrm{~s}$ was used for the short-term analysis and $0.2 \mathrm{~s}$ for the long-term analysis. As the short term analysis involves detailed calculation of oscillation frequencies $(0.05-0.2 \mathrm{~Hz})$ in the region of thermocline, a relatively lower time step of $0.1 \mathrm{~s}$ was considered, which is sufficient to resolve frequencies up to $5 \mathrm{~Hz}$. Convergence was achieved at each time step.

\section{Results}

The axial and radial distances are non-dimensionalized by the tank height and radius, respectively $\left(h^{*}=y / H, r^{*}=2 r / D\right)$. The temperature is non-dimensionalized relative to the hot and cold temperature extremes $\left(T^{*}=\left(T-T_{c}\right) /\left(T_{h}-T_{c}\right)\right)$, while the velocity is non-dimensionalized by the characteristic inlet velocity $\left(u^{*}=u / v_{c}, v^{*}=v / v_{c}\right)$.

Time is non-dimensionalized for the short-term sensitivity analysis in two different ways. For the establishment of an initial and stable thermocline, the non-dimensional time is defined as $\tau_{\text {in }}=t v_{\mathrm{c}} / H$. For the introduction and evolution of the flow disturbance, the time is nondimensionalized by the time scale of the Rayleigh-Taylor instability $\left(\tau^{*}=t / \tau_{\mathrm{c}}\right)$. The time is reset to zero at the beginning of the flow disturbance.

\subsection{Short-term sensitivity to a flow disturbance}

\subsubsection{Initial thermal stratification}

During the charging process, the thermocline region moves axially downward, forcing out cold fluid from the bottom of the tank, as can be observed in the evolution of temperature profiles at the centerline (axis) and side wall shown in Figure 2. When hot salt is flowing inside the tank, the temperature at the side wall is slightly lower than the temperature at the centerline. When the thermocline reaches half the height of the tank $\left(h^{*}=0.5\right)$ the flow is stopped, and the fluid near the side wall is vertically repositioned according to its density by buoyancy forces. At $\tau_{\text {in }}=1.51$, the 
radial temperature gradients caused by flow confinement practically vanish. The maximum temperature difference between the side wall and the axis is less than $2 \%$ of the maximum temperature difference at the end of the half-charge process. A stable, radially uniform thermocline is established at the middle of the tank. The thermocline has thicknesses of $0.18 \mathrm{~m}, 0.14 \mathrm{~m}$, and $0.14 \mathrm{~m}$ for Atwood numbers of $0.00167,0.0084$, and 0.0167 , respectively. In the present analysis, the thermocline region is defined as the zone where the non-dimensional local temperature lies within the range of $0.01 \leq T^{*} \leq 0.99$. Due to stronger buoyancy forces, the thermocline region shrinks with an increase in Atwood number.

\subsubsection{Growth of disturbance}

Temperature contour plots in Figure 3 show the evolution of the disturbance. Under the influence of gravity, the incoming lower-temperature fluid propagates downwards. Rayleigh-Taylor instability causes rolling of the incoming fluid [10]. Consequently, a toroidal vortex is formed near the inlet region. The size of the toroidal vortex increases with time. The advecting vortex morphs into a spike-like structure with increasing downstream distance from the inlet. Similar flow structures have been observed by Manu et al. [10]. The difference in temperature between the disturbance fluid and the hot fluid is kept constant across all three cases. A constant Atwood number $\left(\mathrm{At}_{d}\right)$ of 0.00085 is defined based on the density of the disturbance fluid compared to the density of the hot fluid, both of which are held constant across the cases. The formation and growth of the vortex before interacting with the thermocline is similar for all Atwood numbers as evident from the first three contours of Figure 3. This is expected because $\mathrm{At}_{d}$ uniquely quantifies the evolution of the vortex upstream of the thermocline. The vortex-thermocline interaction becomes predominant at $\tau^{*}=3.1$.

\subsubsection{Vortex-thermocline interaction}

For low values of Atwood number $($ At $=0.00167)$, the stratification is weak. In such cases (Figure $3 a)$, the vortex completely penetrates the thermocline. For a higher Atwood number $($ At $=0.0167)$, the incoming fluid density is sufficiently lower than the cold fluid density. As a result, the disturbance and associated fluid structures encounter resistance at the interface (Figure 3c). Strong stratification retards the motion of the descending spike-like structure. Consequently, the disturbance decays and barely penetrates the thermocline. For these cases, the mixing occurs predominantly in the top half of the thermocline with a relatively undisturbed lower portion (Figure $3 c)$.

Figure 4 shows the variation of penetration length $\left(L_{p}{ }^{*}\right)$ with time. Penetration length is defined at two time intervals: from $\tau^{*}=0$ to $\tau^{*}=3.1$, the axial distance from the top of the tank corresponding to the minimum axial temperature gradient along the centerline is considered as the 
penetration length; however, after $\tau^{*}=3.1$, the penetration length is defined based on the maximum value of the axial temperature gradient. Values of maximum penetration lengths are tabulated in Table 1. The maximum penetration is observed for the lowest Atwood number case. For the relatively higher Atwood numbers, penetration lengths are much lower due to reduced interaction with the thermocline.

\subsubsection{Axial distribution of temperature}

The temperature contour at the last time instant of $\tau^{*}=73$ shown in Figure 3 is indicative of the ultimate effect of the disturbance on the thermocline. For higher Atwood number cases, the region of high axial temperature variation is mainly restricted to the lower half of the tank (Figure 3b-c). For the lowest Atwood number case, a significant temperature reduction is observed over a much larger axial distance. The absolute value of the thermocline thickness, $\delta$, prior to the disturbance differs for each Atwood number case, as discussed in Section 3.1.1. Thermocline thickness values obtained after the evolution of the flow disturbance and the subsequent settling period, and the corresponding percentage increase compared to the initial stratified condition, are listed in Table 1. The thermocline thickness increases by a large percentage for the lowest Atwood number case (74.8\% for $\mathrm{At}=0.00167)$, due to the additional mixing induced by the relatively stronger disturbance (Figure 3a). For the higher Atwood number cases (At $=0.00844$ and At $=0.0167$ ), the percentage change in thermocline thickness values $(13.98 \%$ and $8.97 \%$, respectively) is small due to resiliency against entrainment of disturbance fluid (Figure 3b-c).

\subsubsection{Radial distribution of temperature}

Vortex-thermocline interaction starts predominantly in the center-axis region of the tank and propagates in the radial direction towards the side walls. The radial distribution of temperature along the non-dimensional thickness of the thermocline $\left(\delta^{*}=\delta / H\right)$ at different instants after the flow disturbance introduction has stopped, in Figures 5, 6, and 7, shows the spatio-temporal features. The middle of the thermocline in all three cases is located at $h^{*}=0.31$. The temporal variations of the radial temperature distributions are plotted at three different positions $\left(h^{*}=0.31+\delta^{*} / 2\right.$, and 0.31 $\delta^{*} / 2$ ) along the thickness of the thermocline.

For the lowest Atwood number case $(\mathrm{At}=0.00167)$ (Figure 5), the temperature profiles exhibit strong oscillations that amplify with time, indicating strong vortex-thermocline interaction. The temperature perturbation amplitudes are initially largest at the central axis (Figure 5) and propagate in the radial direction with time. The effects of the disturbance are observed at progressively later times (phase lags) along the depth of the thermocline (Figure 5a-c). On the other hand, for higher Atwood number cases (At $=0.00844$ and At $=0.0167)$, the disturbance is unable to influence the temperature in the lower part of the thermocline (Figures 6 and $7 ; h^{*}=0.31-\delta^{*} / 2$ ). 
The disturbance first deforms the thermocline region, followed by entrainment/mixing of cold fluid. The flow time instants corresponding to the inception of deformation and entrainment/mixing are denoted as $\tau_{\delta o}^{*}$ and $\tau_{m o}^{*}$, respectively. These values are determined by observing the variation of $\delta^{*}$ and $T_{m}{ }^{*}$ along the radial direction. The time lag between deformation and mixing $\left(\tau_{\mathrm{lag}}\right)$ is calculated by measuring the difference between these times. The values of $\tau_{\delta o}^{*}$ are identical across all Atwood number cases because the disturbance and hot fluid temperatures are fixed. However, the mixing process starts at different time instances due to increased resistance to the disturbance at the interface with increasing Atwood number. The values of $\tau_{\delta o}^{*}, \tau_{m o}^{*}$ and $\tau_{\text {lag }}$ are summarized in Table 2. The value of $\tau_{m o}^{*}$ increases with increasing Atwood number. For low Atwood numbers $(A t=0.00167)$, the restoring buoyancy forces are weak due to the mild density gradient across the thermocline region, resulting in the low value of $\tau_{m o}^{*}$.

The radial variation of thermocline thickness is calculated for all three Atwood number cases, and shown in Figure 8 at different time instants. The change in thermocline thickness is larger near the center axis as compared to the near-wall region. In the vicinity of the side walls, variations of thermocline thickness due to the fluid structure-thermocline interaction are insignificant. The mean temperature inside the thermocline region $\left(T_{m}{ }^{*}\right)$, averaged along the axial direction, is calculated. The entrainment process causes a span-wise variation of $T_{m}{ }^{*}$. Figure 9 shows the radial variation of $T_{m}{ }^{*}$ in the thermocline region. It is further evident that the initial stage of mixing is dominant in the center-axis region, with insignificant change in $T_{m}{ }^{*}$ in the near-wall region.

\subsubsection{Thermocline response}

Fluid structure-density gradient interaction creates internal waves near the interface. As a result of vortical interactions, periodic oscillations are detected inside the thermocline region. Axial velocity is monitored at a fixed position $\left(r^{*}=0, h^{*}=0.32\right)$ for all Atwood number cases, and is shown in Figure 10. The amplitude of the velocity oscillations decays with time as restoring buoyancy forces dampen the oscillations of the thermocline. A damping time constant $\left(\tau_{\text {damp }}^{*}\right)$ is calculated by measuring the time taken for the amplitude of the axial velocity signal at this fixed position to decay to $1 \%$ of its initial value. The quantitative details of the under-damped oscillations are provided in Table 3. For At $=0.00167$, restoring buoyancy forces are weak, resulting in the highest damping time $\left(\tau_{\text {damp }}^{*}=26.02\right)$. For higher Atwood numbers, relatively stronger buoyancy forces dampen the oscillations faster $\left(\tau_{\text {damp }}^{*}=12.78\right.$, for At $=0.00844$ and $\tau_{\text {damp }}{ }^{*}=8.22$ for At $\left.=0.01167\right)$.

The velocity at the fixed position noted above oscillates with different frequencies. The maximum permitted oscillation frequency due to the density gradient in a stratified medium 
corresponds to Brunt-Väisälä frequency $\left(N=(-g / \rho(\mathrm{d} \rho / \mathrm{d} y))^{1 / 2}\right)$. The power spectral density profiles for all three Atwood numbers shown in Figure 11 indicate the dominant oscillation frequencies. The power density is largest at low frequencies that are less than the Brunt-Väisälä frequency. Similar behavior was observed in case of successive multi-vortex interaction (with different vortex generation mechanism) with thermocline [21]. The peak frequency values and the Brunt-Väisälä frequency values, tabulated in Table 3, increase with Atwood number.

\subsection{Long-term sensitivity to a flow disturbance}

\subsubsection{Time-periodic response}

Under cyclical operation of the thermocline tank, a fraction of the thermocline region is always retained inside the tank; hence, the thermocline region extent is limited between a minimum and maximum bound for the time-periodic response. The thermocline region achieves its maximum extent towards the end of the charging or discharging processes, as it approaches the outlet port. As part of the thermocline exits the tank, its extent reduces until it achieves a minimum value just at the end of the charging $\left(t=t_{0}\right)$ or the discharging process $\left(t=2 t_{0}\right)$. When the flow is reversed, the thermocline starts growing again. Wall effects cause deformation of the thermocline region from an ideal cylindrical plug, and it is necessary to define an effective thermocline thickness as the ratio between the volume occupied by the thermocline region and the cross-sectional area. The effective thermocline thickness under time-periodic response for the different Atwood numbers at an instant close to the end of the charging process $\left(t=0.75 t_{0}\right)$ is presented in Table 4 . Similar trends as for the charging process of an isothermal tank are observed, exhibiting a decrease in the effective thermocline thickness as the Atwood number increases with values of $0.34 \mathrm{~m}, 0.27 \mathrm{~m}$, and $0.26 \mathrm{~m}$ for Atwood numbers of 0.00167, 0.0084, and 0.0167, respectively.

The overall thermal performance under time-periodic response presents a trend with respect to Atwood number that is in agreement with the previous observations. The storage efficiency based on a first-law balance is an appropriate metric of the thermal performance and it is defined as follows:

$$
\eta_{I}=\frac{\int_{t_{0}}^{2 t_{o}}\left[\dot{m} c_{p}\left(\bar{T}-T_{c}\right)\right]_{y=H}}{\int_{0}^{t_{o}}\left[-\dot{m} c_{p}\left(\bar{T}-T_{c}\right)\right]_{y=H}}
$$


Despite the wide range in the Atwood numbers considered, high storage efficiencies (Table 5) are obtained under time-periodic response for all three cases. For Atwood numbers of 0.00844 and 0.0167, the thermocline tank achieves identical values of storage efficiency of $98.8 \%$. For the lowest Atwood number of 0.00167 , the efficiency is marginally lower $(98.5 \%)$.

\subsubsection{Thermal stratification recovery after disturbance}

Figure 12 presents temperature contours inside the thermocline tank at two instants during the charging-discharging cycles for the time-periodic response, during the cycle with a flow disturbance, and during the cycle immediately following the disturbance for the three Atwood numbers considered. The flow disturbance drastically affects the temperature distribution inside the thermocline tank during the cycle in which it is applied, and flow structures similar to those from the short-term study are obtained. However, the thermal stratification inside the tank quickly recovers to the temperature distribution of the time-periodic response in the following cycle in each case.

The effective thermocline thickness confirms the observations from the temperature contours (Table 4). The recovery of thermal stratification is slightly less effective as the Atwood number decreases, and some minor differences in the temperature distribution, and a marginally thicker thermocline, when compared to the time-periodic response, are obtained for the case of $\mathrm{At}=0.00167$ in the cycle immediately after the flow disturbance is applied.

\subsubsection{Disturbance effect on thermal storage efficiencyError! Reference source not}

found.Figure 13 shows the effect of the flow disturbance on the discharge temperature of the labscale thermocline tank geometry considered in this work. During the discharge process of the cycle with a flow disturbance, the outflow temperature markedly drops as a result of the non-ideal charging profile. However, the temperature outflow is almost completely restored in the cycle immediately following the disturbance. For this subsequent cycle, the discharge temperature is only lower $\left(\Delta T^{*}{ }_{\max }=0.0215\right)$ than the time-periodic response for the lowest Atwood number case of At $=0.00167$.

The first-law efficiency also confirms the resilience of the thermocline tank to the flow disturbance in the long term (Table 5). The efficiency drops during the cycle which experiences the disturbance, but quickly recovers in the following cycle. The decrease in efficiency is more pronounced for the case with low Atwood number. Also, for this case the performance recovery is slightly slower.

\section{Conclusion}


Numerical simulations are presented for the charge-discharge processes in a stratified thermocline tank. The short-term spatio-temporal effects on the thermocline, as well as long-term effects on subsequent cyclic charging-discharging of the tank resulting from the introduction of a flow disturbance are assessed at different Atwood numbers.

In the short-term analysis, the disturbance propagates downstream and interacts with the thermal stratification layer inside the TES tank. The penetration of a given disturbance into the thermocline layer and the thermocline thickness increase with decreasing Atwood number. During the disturbance-thermocline interactions, a time lag between the initial deformation of the thermocline and the subsequent mixing process is observed; this time lag increases with Atwood number. Vortex-thermocline interaction also leads to spatio-temporal fluctuations of temperature and stratification thickness; a power spectrum analysis indicates the presence of low-frequency peaks that are lower than the Brunt-Väisälä frequency.

In the long term, simulations of a charging-discharging cycle with a flow disturbance revealed that the outflow temperature during discharge is reduced (relative to the time-periodic behavior without a disturbance) due to mixing caused by the disturbance. However, the thermocline temperature profile is restored to the time-periodic state in the subsequent charging-discharging cycle. If appropriately designed and operated to ensure high Atwood numbers during flow disturbance events, thermocline tanks can thus be a robust energy storage technology for CSP applications.

\section{Acknowledgement}

This paper is based upon work supported in part by the India-US partnership to Advance Clean Energy-Research (PACE-R) for the Solar Energy Research Institute for India and the United States (SERIIUS), funded jointly by the U. S. Department of Energy (Office of Science, Office of Basic Energy Sciences, and Energy Efficiency and Renewable Energy, Solar Energy Technology Program, under Subcontract DE-AC36-08GO28308 to the National Renewable Energy Laboratory, Golden, Colorado) and the Government of India, through the Department of Science and Technology under Subcontract IUSSTF/JCERDC-SERIIUS/2012 dated 22nd Nov. 2012 and DSTSERI-Project No: DST/TMC/SERI/FR/136. CMH acknowledges financial support from the

Colombia-Purdue Institute (CPI) and the Colombian department for science, technology and innovation (Colciencias).

\section{References}

[1] International Energy Agency, Technology Roadmap: Concentrating Solar Power, 2010.

[2] A. Gil, M. Medrano, I. Martorell, A. Lázaro, P. Dolado, B. Zalba, L.F. Cabeza, State of the 
art on high temperature thermal energy storage for power generation. Part 1-Concepts, materials and modellization, Renew. Sustain. Energy Rev. 14 (2010) 31-55.

[3] J.E. Pacheco, M.E. Ralph, J.M. Chavez, S.R. Dunkin, E.E. Rush, C.M. Ghanbari, M.W. Matthews, Results of molten salt panel and component experiments for solar central receivers: cold fill, freeze/thaw, thermal cycling and shock, and instrumentation, Sandia Natl. Lab. Rep. No. SAND94-2525. (1995).

[4] S.M. Flueckiger, Z. Yang, S. V. Garimella, Review of Molten-Salt Thermocline Tank Modeling for Solar Thermal Energy Storage, Heat Transf. Eng. 34 (2013) 787-800.

[5] J.E. Pacheco, S.K. Showalter, W.J. Kolb, Development of a molten-salt thermocline thermal storage system for parabolic trough plants, J. Sol. Energy Eng. 124 (2002) 153-159.

[6] S.M. Flueckiger, B.D. Iverson, S. V. Garimella, Economic Optimization of a Concentrating Solar Power Plant With Molten-Salt Thermocline Storage, J. Sol. Energy Eng. 136 (2013) 011016.

[7] F.G.F. Qin, X. Yang, Z. Ding, Y. Zuo, Y. Shao, R. Jiang, X. Yang, Thermocline stability criterions in single-tanks of molten salt thermal energy storage, Appl. Energy. 97 (2012) 816-821.

[8] Z. Yang, S. V. Garimella, Thermal analysis of solar thermal energy storage in a molten-salt thermocline, Sol. Energy. 84 (2010) 974-985.

[9] M.Y. Haller, C.A. Cruickshank, W. Streicher, S.J. Harrison, E. Andersen, S. Furbo, Methods to determine stratification efficiency of thermal energy storage processes - Review and theoretical comparison, Sol. Energy. 83 (2009) 1847-1860.

[10] K. V. Manu, P. Anand, U.K. Chetia, S. Basu, Effects of instabilities and coherent structures on the performance of a thermocline based thermal energy storage, Appl. Therm. Eng. 87 (2015) 768-778.

[11] K.V. Manu, P. Deshmukh, S. Basu, Rayleigh-Taylor instability in a thermocline based thermal storage tank, Int. J. Therm. Sci. 100 (2016) 333-345.

[12] P.F. Linden, The interaction of a vortex ring with a sharp density interface: a model for turbulent entrainment, J. Fluid Mech. 60 (1973) 467-480.

[13] W.J.A. Dahm, C.M. Scheil, G. Tryggvason, Dynamics of vortex interaction with a density interface, J. Fluid Mech. 205 (1989) 1-43.

[14] D.J. Tritton, Physical fluid dynamics, 2nd ed., Oxford University Press, New York, 1988.

[15] Y. Noh, H. Fernando, C. Ching, Flows induced by the impingement of a two-dimensional thermal on a density interface, J. Phys. Oceanogr. 22 (1992) 1207-1220.

[16] E. Kaloudis, D.G.E. Grigoriadis, E. Papanicolaou, T. Panidis, Large eddy simulation of thermocline flow phenomena and mixing during discharging of an initially homogeneous or stratified storage tank, Eur. J. Mech. B/Fluids. 48 (2014) 94-114. 
[17] Z. Yang, S. V. Garimella, Cyclic operation of molten-salt thermal energy storage in thermoclines for solar power plants, Appl. Energy. 103 (2013) 256-265.

[18] S.M. Flueckiger, B.D. Iverson, S. V. Garimella, J.E. Pacheco, System-level simulation of a solar power tower plant with thermocline thermal energy storage, Appl. Energy. 113 (2014) 86-96.

[19] ANSYS, Fluent 15.7.0, (2014).

[20] R.I. Issa, Solution of the implicitly discretised fluid flow equations by operator-splitting, J. Comput. Phys. 62 (1986) 40-65.

[21] Aashay Tinaikar, Advaith S, Utpal Kumar Chetia, Manu K V and Saptarshi Basu, Spatiotemporal Disruption of Thermocline by Successive Laminar Vortex Pairs in a Single Tank Thermal Energy Storage, Appl. Therm. Eng. DOI: 10.1016/j.applthermaleng.2016.04.105 


\section{Tables}

Table 1 Vortex-thermocline interaction parameters.

\begin{tabular}{|c|c|c|c|c|c|}
\hline $\begin{array}{c}\text { Atwood } \\
\text { Number } \\
\text { At }\end{array}$ & $\begin{array}{c}\text { Non-dimensional max. } \\
\text { penetration length } \\
L_{p}^{*}\end{array}$ & $\begin{array}{c}\text { Non-dimensional time of } \\
\text { max. penetration } \\
\tau^{*}\end{array}$ & \multicolumn{3}{|c|}{$\begin{array}{c}\text { Non-dimensional thermocline } \\
\text { thickness, } \\
\delta^{*}\end{array}$} \\
\cline { 4 - 6 } & & & $\tau^{*}=0.0$ & $\tau^{*}=73.0$ & $\%$ Change \\
\hline 0.00167 & 0.82 & 3.74 & 0.18 & 0.31 & $74.9 \%$ \\
\hline 0.00844 & 0.71 & 3.37 & 0.14 & 0.16 & $14.0 \%$ \\
\hline 0.0167 & 0.69 & 3.28 & 0.14 & 0.15 & $8.6 \%$ \\
\hline
\end{tabular}

Table 2 Time instants of inception of thermocline deformation and mixing.

\begin{tabular}{|c|c|c|c|}
\hline $\begin{array}{c}\text { Atwood } \\
\text { Number } \\
\text { At }\end{array}$ & $\begin{array}{c}\text { Non-dimensional time of } \\
\text { inception of deformation, } \\
\tau_{\delta o}^{*}\end{array}$ & $\begin{array}{c}\text { Non-dimensional time } \\
\text { of inception of mixing, } \\
\tau_{m o}^{*}\end{array}$ & $\begin{array}{c}\text { Non-dimensional time lag between } \\
\text { inception of deformation and mixing, } \\
\tau_{\text {lag }}^{*}=\tau_{m o}^{*}-\tau_{\delta o}^{*}\end{array}$ \\
\hline 0.00167 & 2.20 & 2.37 & 0.17 \\
\hline 0.00844 & 2.20 & 2.42 & 0.22 \\
\hline 0.0167 & 2.20 & 2.43 & 0.23 \\
\hline
\end{tabular}

Table 3 Parameters of under-damped oscillations induced by flow disturbance at $r^{*}=0, h^{*}=0.32$.

\begin{tabular}{|c|c|c|c|}
\hline $\begin{array}{c}\text { Atwood } \\
\text { At }\end{array}$ & $\begin{array}{c}\text { Brunt-Väisälä } \\
\text { frequency } \\
N(\mathrm{~Hz})\end{array}$ & $\begin{array}{c}\text { Peak } \\
\text { frequency } \\
(\mathrm{Hz})\end{array}$ & $\begin{array}{c}\text { Non-dimensional damping } \\
\text { constant } \\
\tau_{\text {damp }}^{*}(-)\end{array}$ \\
\hline 0.00167 & 0.1 & 0.02 & 26.02 \\
\hline 0.00844 & 0.25 & 0.12 & 12.78 \\
\hline 0.0167 & 0.35 & 0.17 & 08.22 \\
\hline
\end{tabular}

Table 4 Effective non-dimensional thermocline thickness $\left(\delta^{*}\right)$ at $t / t_{0}=0.75$.

\begin{tabular}{|c|c|c|c|c|c|}
\hline $\begin{array}{c}\text { Atwood } \\
\text { Number } \\
\text { At }\end{array}$ & $\begin{array}{c}\text { Time-periodic } \\
\text { response }\end{array}$ & $\begin{array}{c}\text { Cycle with flow } \\
\text { disturbance }\end{array}$ & $\begin{array}{c}\text { First recovery } \\
\text { cycle }\end{array}$ & $\begin{array}{c}\text { Second } \\
\text { recovery cycle }\end{array}$ & $\begin{array}{c}\text { Third recovery } \\
\text { cycle }\end{array}$ \\
\hline 0.00167 & 0.34 & 0.84 & 0.35 & 0.34 & 0.34 \\
\hline 0.00844 & 0.27 & 0.75 & 0.27 & 0.27 & 0.27 \\
\hline 0.0167 & 0.26 & 0.72 & 0.26 & 0.27 & 0.27 \\
\hline
\end{tabular}

Table 5 First-law efficiency $\left(\eta_{I}\right)$.

\begin{tabular}{|c|c|c|c|c|c|}
\hline $\begin{array}{c}\text { Atwood } \\
\text { number } \\
\text { At }\end{array}$ & $\begin{array}{c}\text { Time-periodic } \\
\text { response }\end{array}$ & $\begin{array}{c}\text { Cycle with flow } \\
\text { disturbance }\end{array}$ & $\begin{array}{c}\text { First recovery } \\
\text { cycle }\end{array}$ & $\begin{array}{c}\text { Second } \\
\text { recovery cycle }\end{array}$ & $\begin{array}{c}\text { Third } \\
\text { recovery cycle }\end{array}$ \\
\hline 0.00167 & $98.5 \%$ & $97.4 \%$ & $98.3 \%$ & $98.5 \%$ & $98.5 \%$ \\
\hline 0.00844 & $98.8 \%$ & $98.4 \%$ & $98.9 \%$ & $98.8 \%$ & $98.8 \%$ \\
\hline 0.0167 & $98.8 \%$ & $98.6 \%$ & $98.9 \%$ & $98.8 \%$ & $98.8 \%$ \\
\hline
\end{tabular}




\section{Figures}
(a) Charging
(b) Disturbance evolution
(c) Charging - Discharging
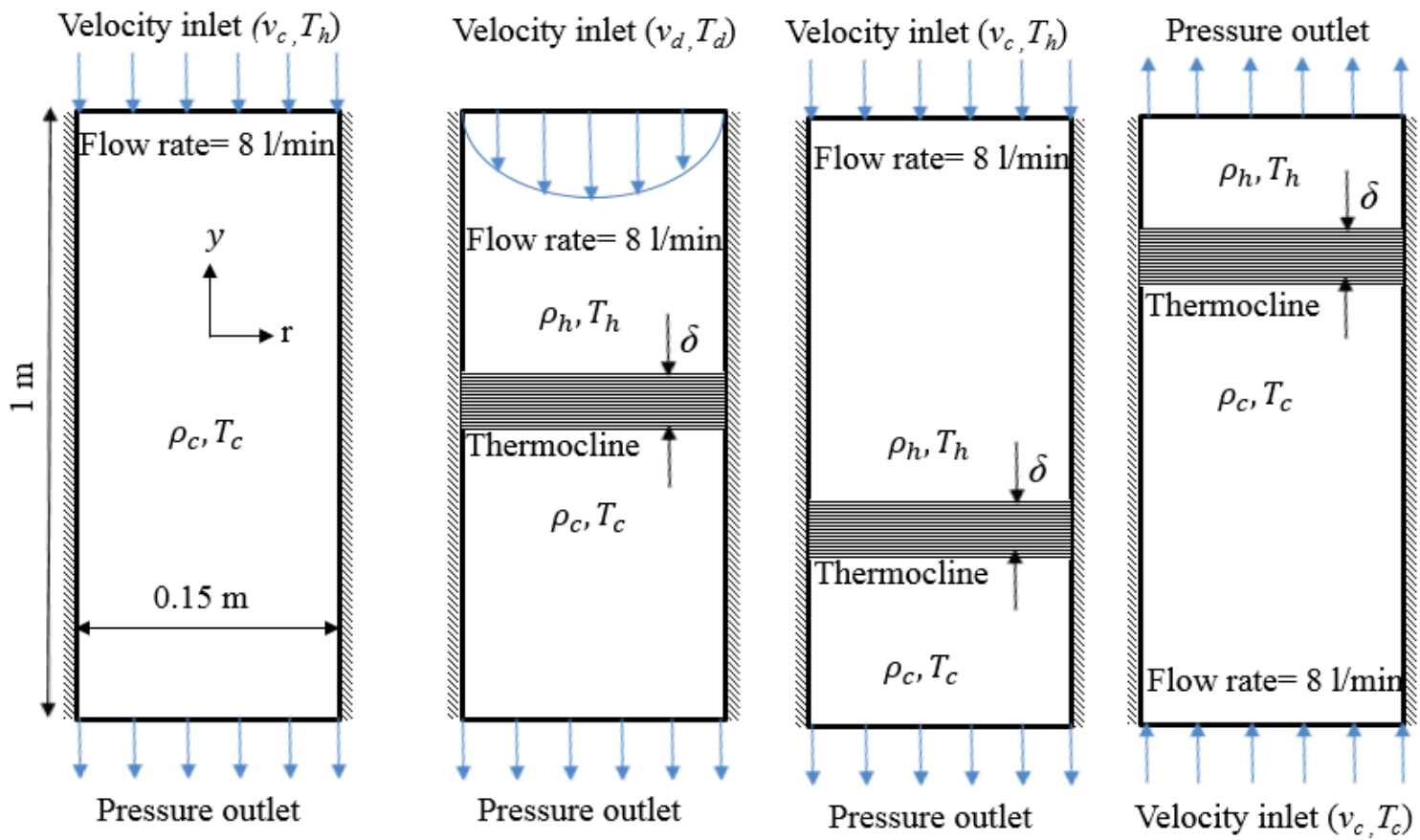

(d) Charging-discharging with disturbance
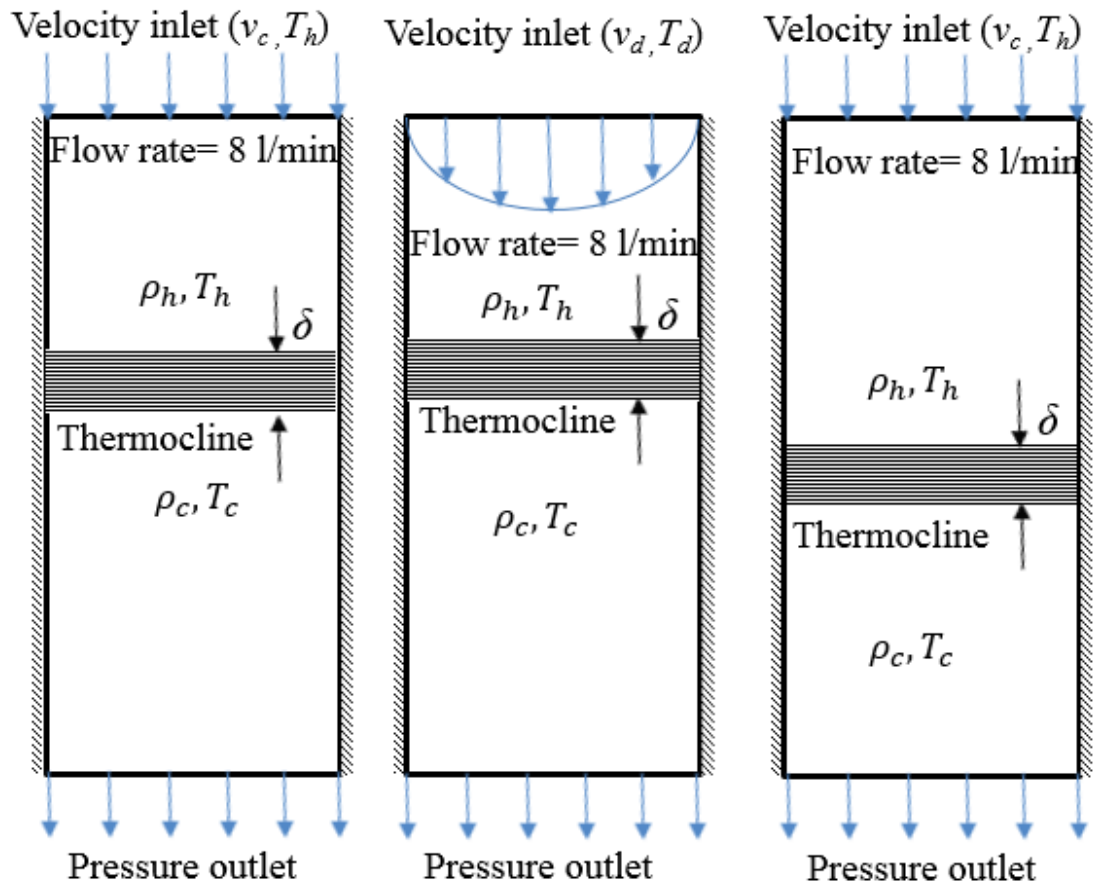

Pressure outlet

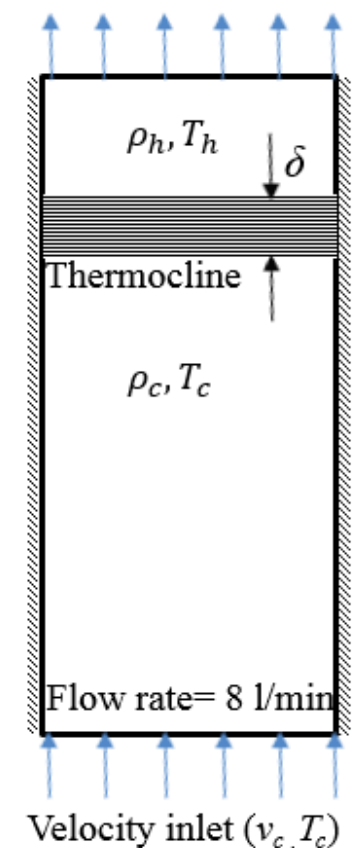

Figure 1: Boundary conditions schematic diagrams: the (a) charging process and (b) introduction and evolution of a disturbance for the short-term analysis; and the (c) charging-discharging cycles without disturbance and (d) charging-discharging cycle with a disturbance for the long-term analysis. The side walls are adiabatic. 

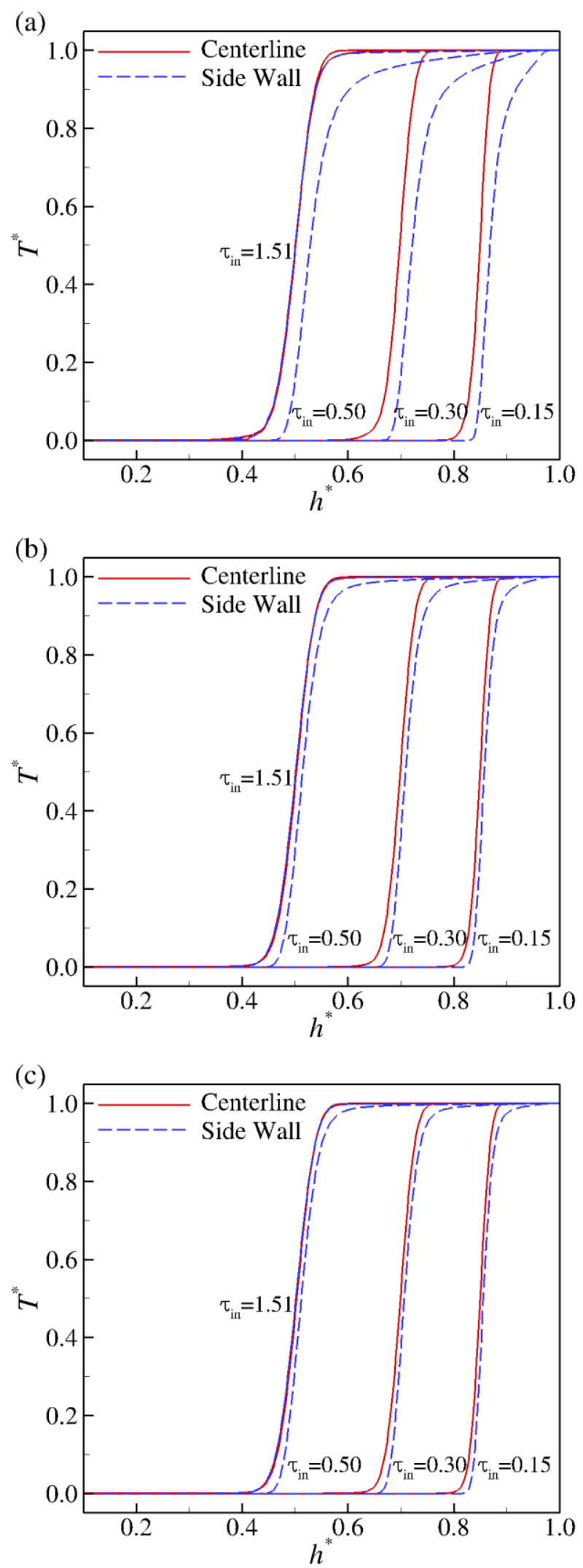

Figure 2: Centerline and wall temperature distribution during the charging of an initially cold tank: (a) At $=0.00167$, (b) At $=0.00844$, and (c) At $=0.0167$. 

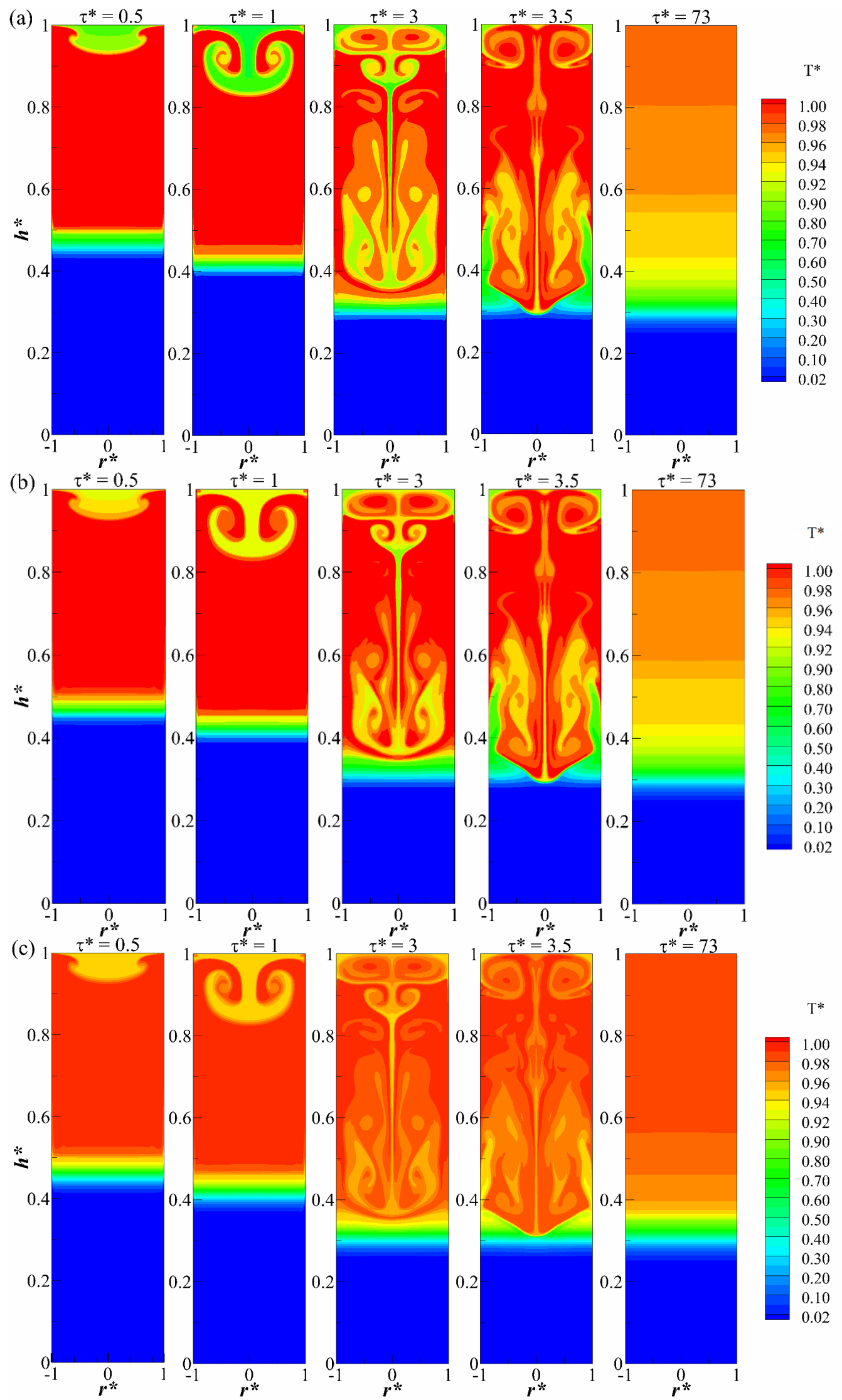

Figure 3: Temperature contours showing the time evolution of the disturbance at selected time instants: (a) At $=0.00167$, (b) At $=0.00844$, and (c) At $=0.0167$. 


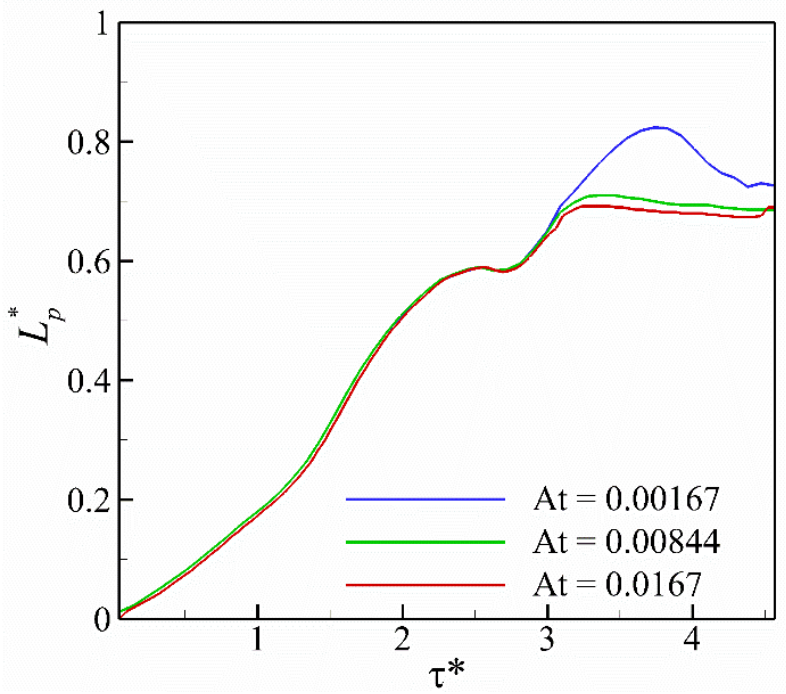

Figure 4: Variation of penetration length of the vortex with time. 
(a)

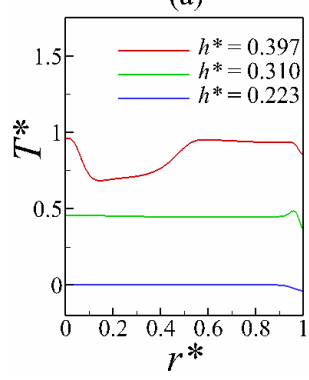

(b)

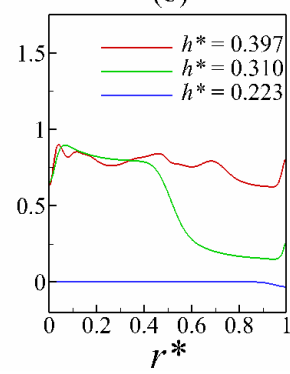

(c)

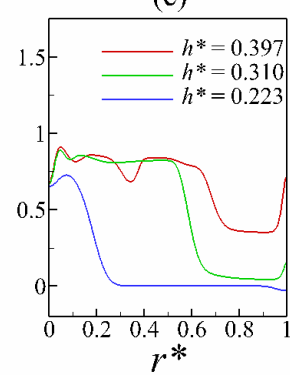

(d)

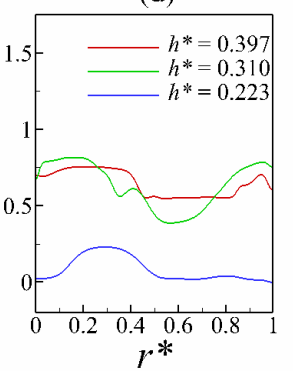

(e)

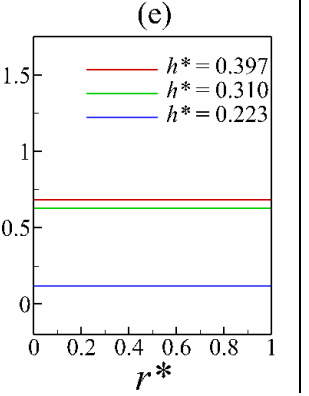

Figure 5: Radial distribution of temperature for At $=0.00167$ at selected tank heights $h^{*}=0.397$, $0.310,0.223$, corresponding to the top, middle, and bottom layer of the thermocline, respectively, after flow disturbance introduction has stopped: (a) $\tau^{*}=2.74$, (b) $\tau^{*}=3.10$, (c) $\tau^{*}=3.28$, (d) $\tau^{*}=5.48$, and $(\mathrm{e}) \tau^{*}=73$. 
(a)

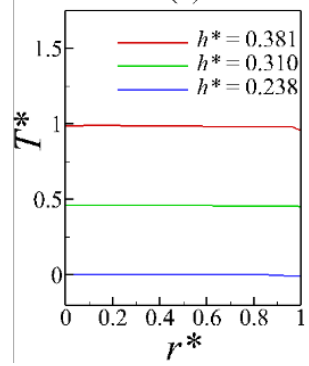

(b)

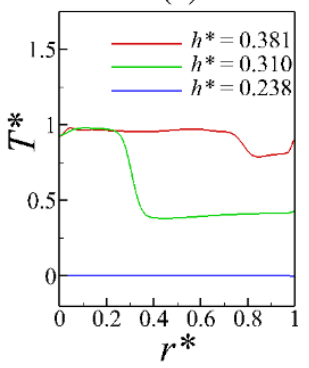

(c)

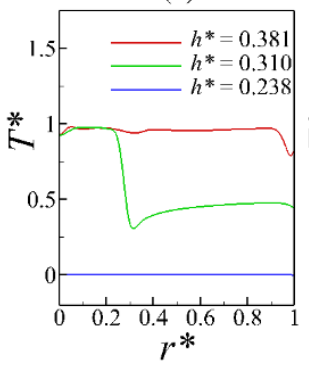

(d)

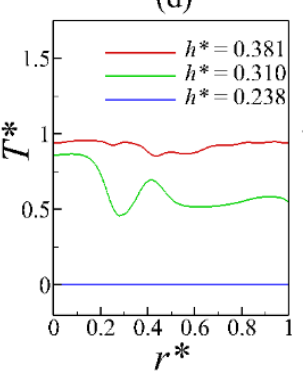

(e)

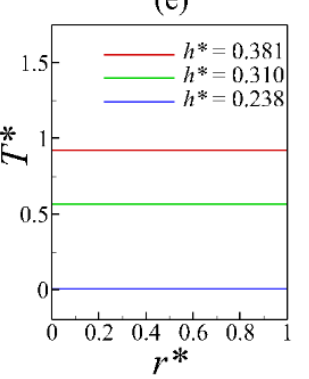

Figure 6: Radial distribution of temperature for At $=0.00844$ at selected heights $h^{*}=0.381,0.310$, 0.238 corresponding to the top, middle, and bottom layer of the thermocline respectively, after flow disturbance introduction has stopped: (a) $\tau^{*}=2.74$, (b) $\tau^{*}=3.10$, (c) $\tau^{*}=3.28$, (d) $\tau^{*}=5.48$, and (e) $\tau^{*}=73$. 
(a)

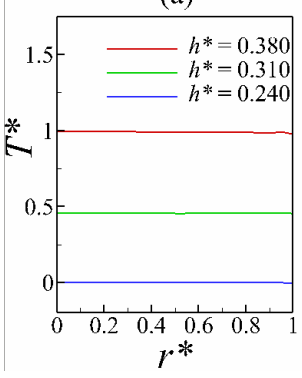

(b)

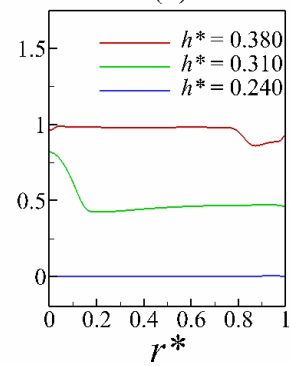

(c)

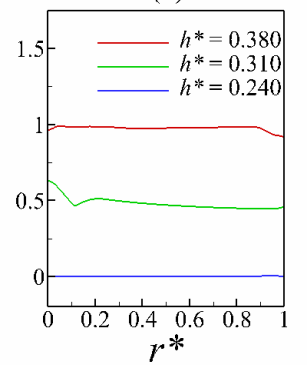

(d)

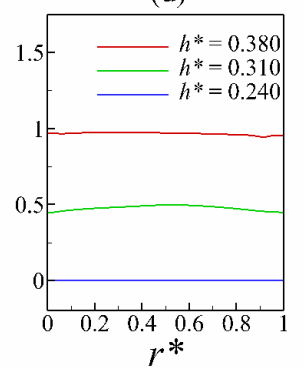

(e)

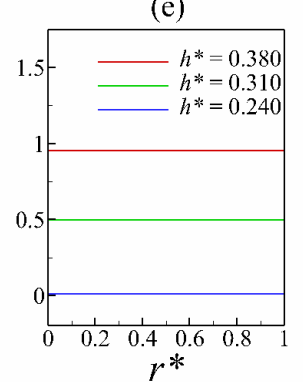

Figure 7: Radial distribution of temperature for At $=0.0167$ at selected heights $h^{*}=0.380,0.310$, 0.240 corresponding to the top, middle, and bottom layer of the thermocline respectively, after flow disturbance introduction has stopped: (a) $\tau^{*}=2.74$, (b) $\tau^{*}=3.10$, (c) $\tau^{*}=3.28$, (d) $\tau^{*}=5.48$, and (e) $\tau^{*}=73$. 

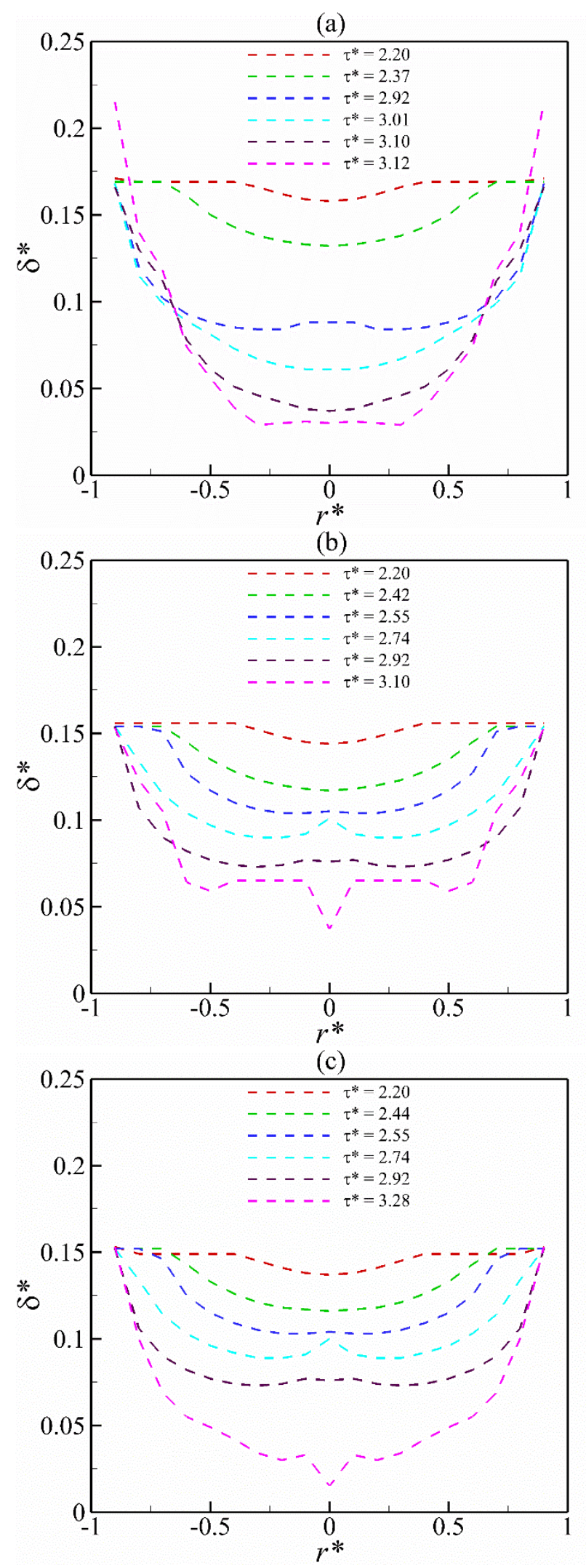

Figure 8: Radial variation of thermocline thickness at selected time instants for: (a) At $=0.00167$, (b) At $=0.00844$, and (c) At $=0.0167$. 

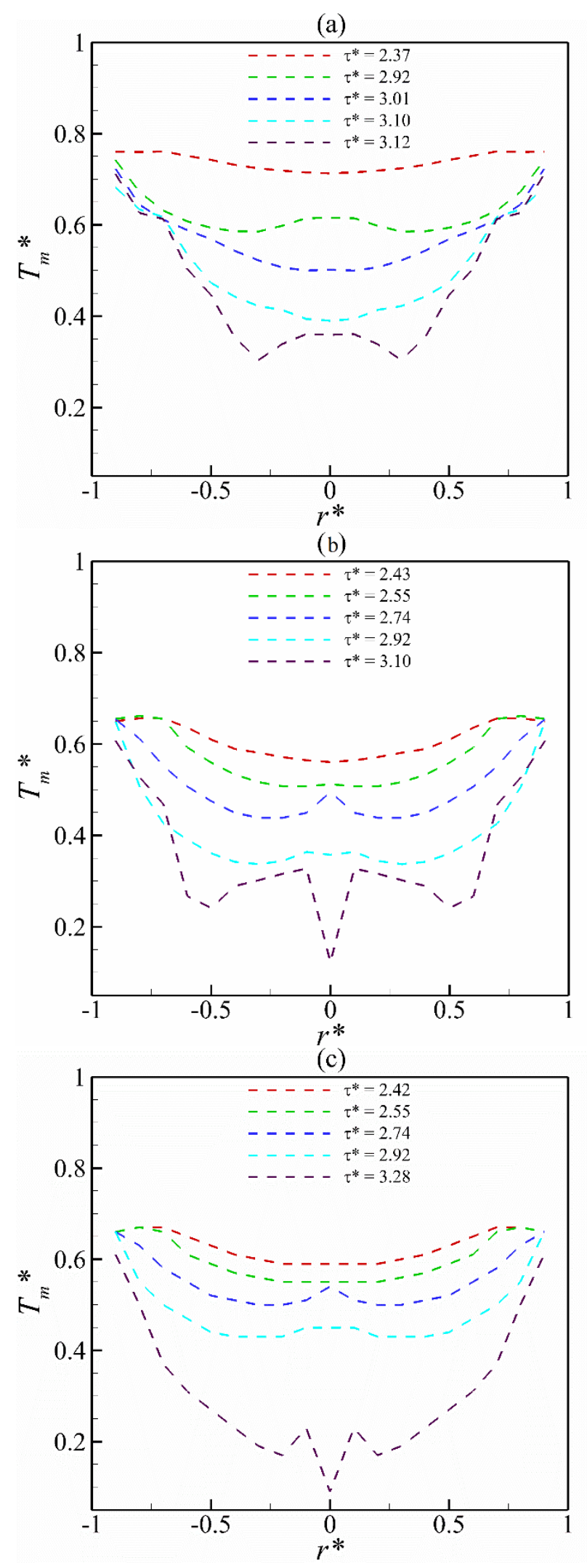

Figure 9: Radial variation of mean temperature calculated in the thermocline region at selected time instants for: (a) At $=0.00167$, (b) At $=0.00844$, and (c) At $=0.0167$. 

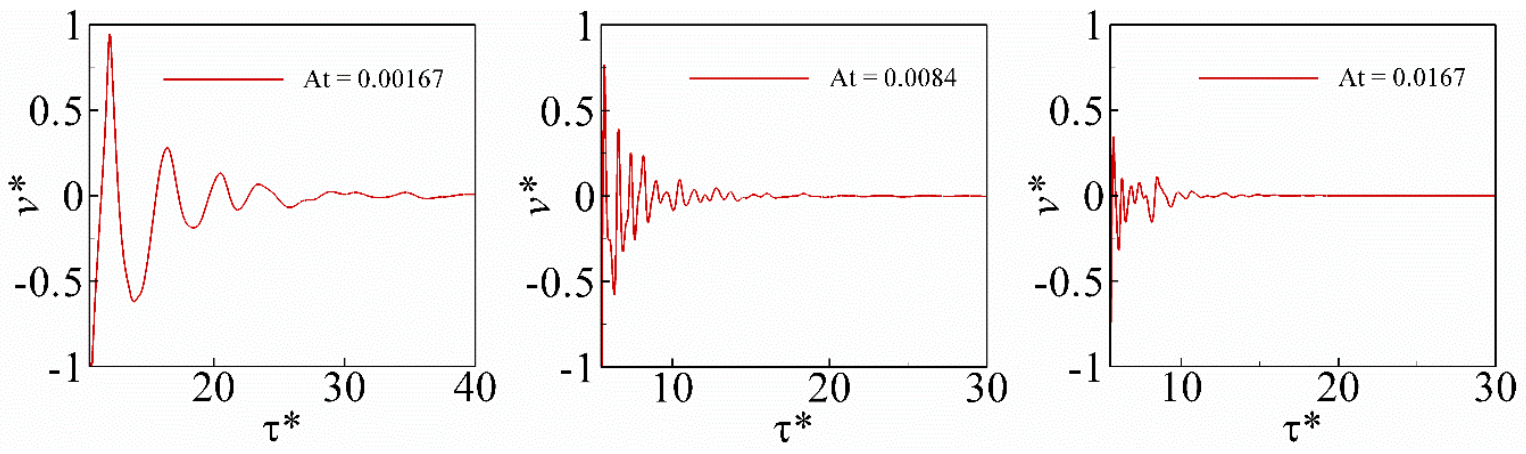

Figure 10: Variation of axial velocity with time at a fixed location $\left(r^{*}=0, h^{*}=0.32\right)$. 

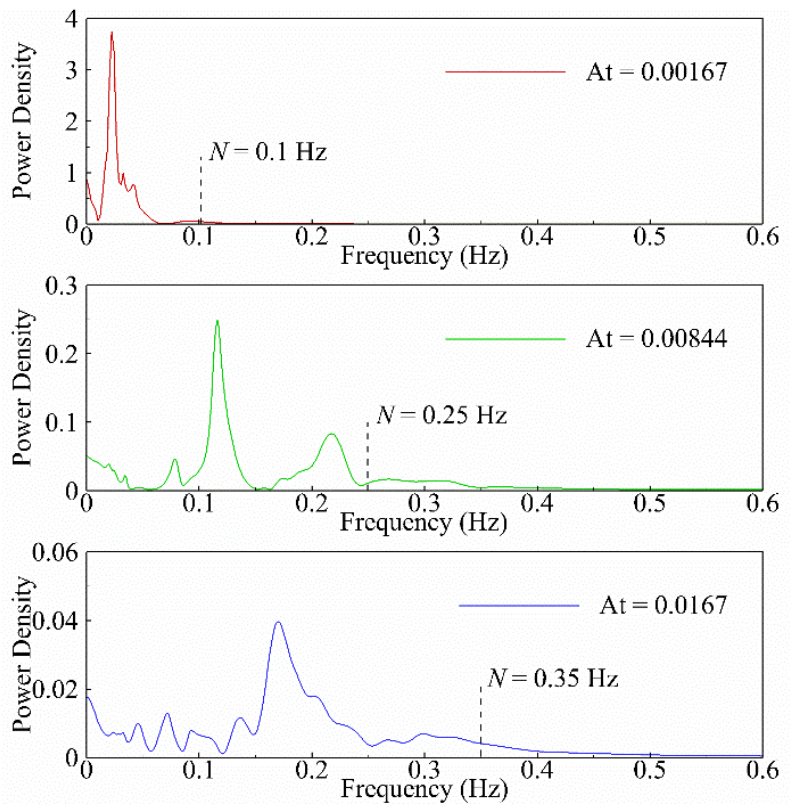

Figure 11: Power spectral density of axial velocity at a fixed location $\left(r^{*}=0, h^{*}=0.32\right)$. 

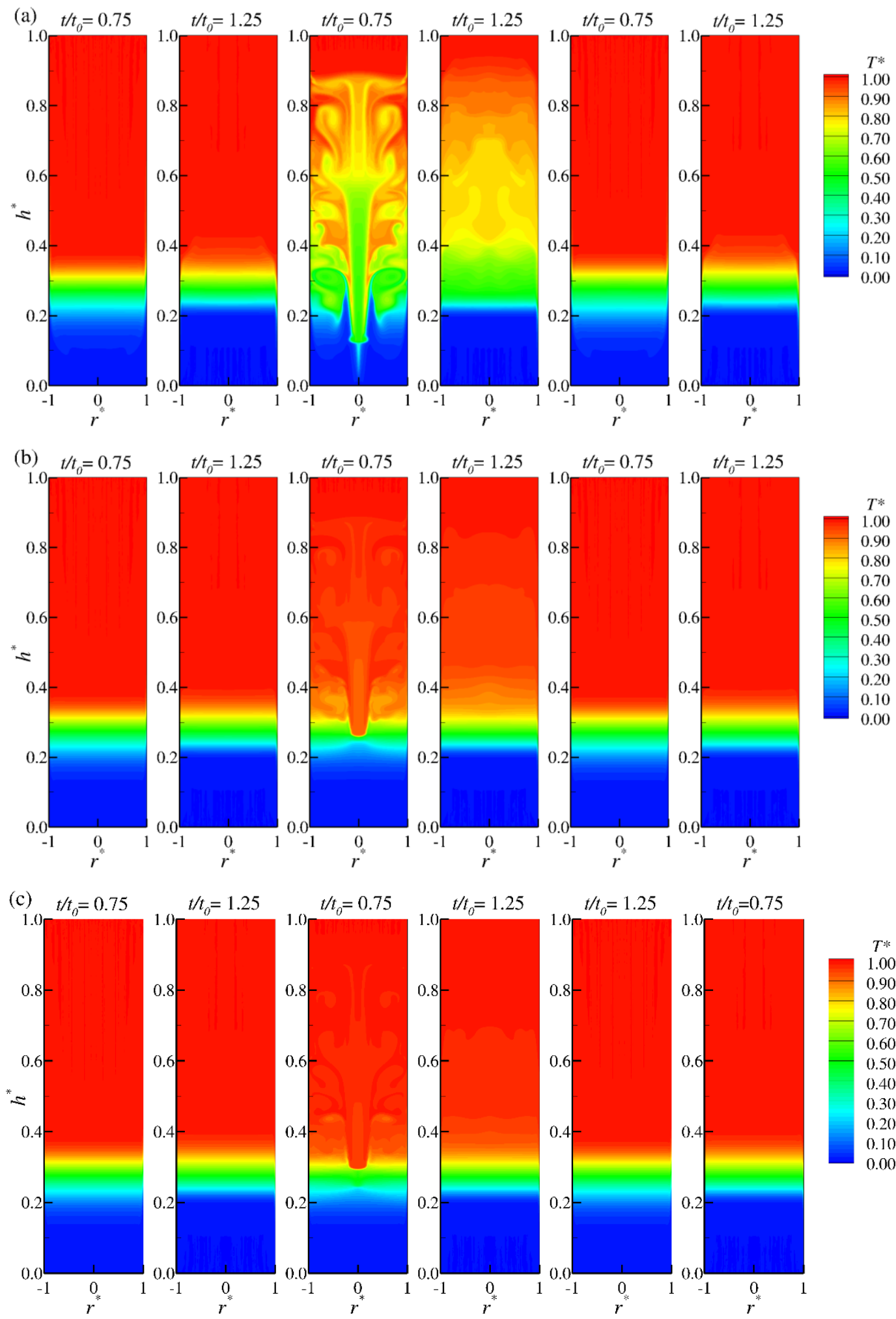

Time-periodic response
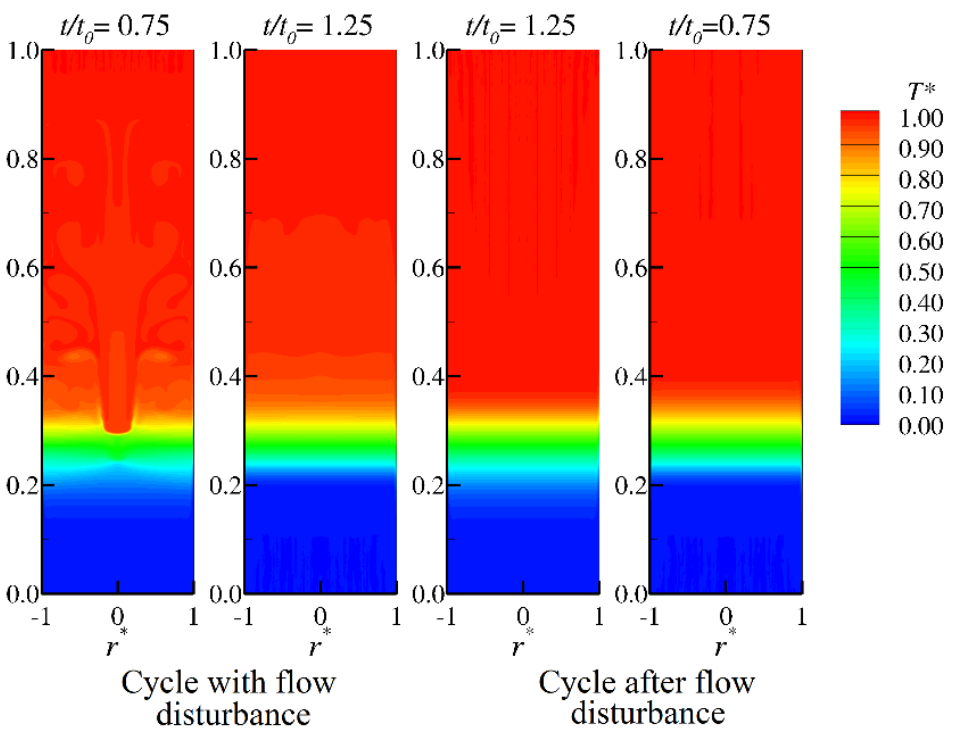

Figure 12: Temperature contours inside the thermocline tank at the same point of the charging $\left(t / t_{0}=0.75\right)$ and discharging $\left(t / t_{0}=1.25\right)$ period before, during, and after the introduction of a disturbance: (a) At $=0.00167$, (b) At $=0.00844$, and (c) At $=0.0167$. 

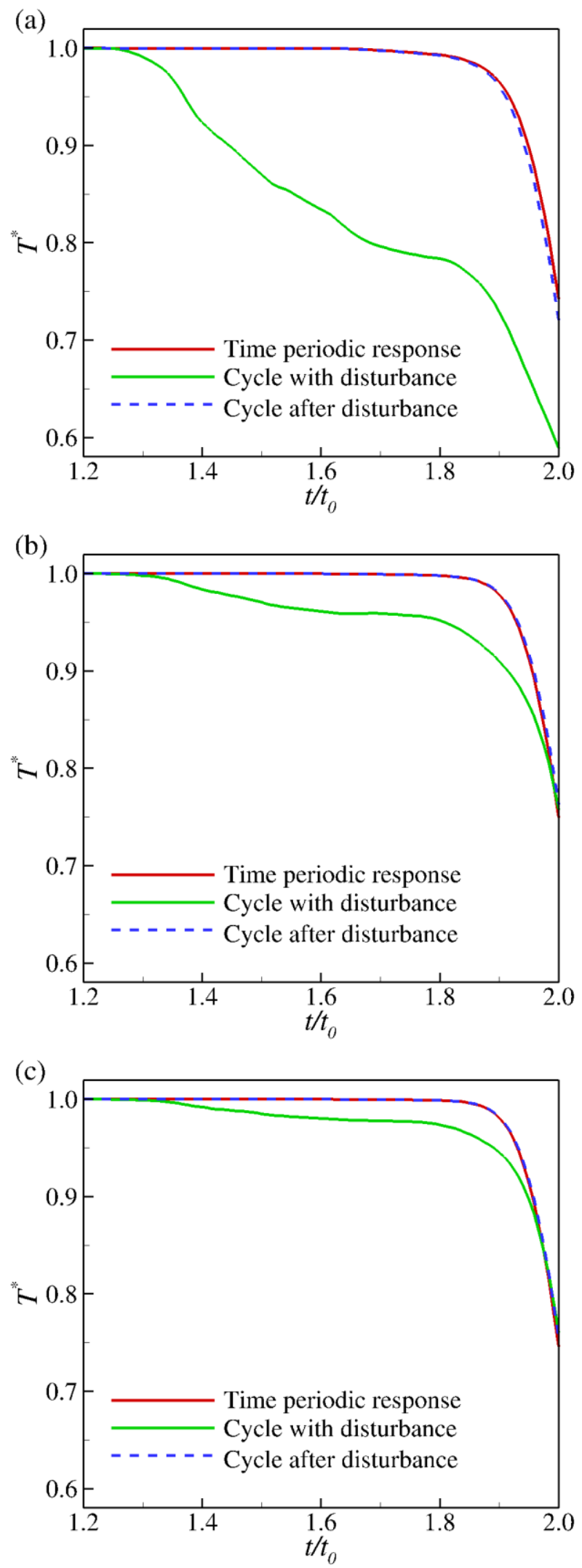

Figure 13: Outflow temperature history during the discharge cycle before, during, and after the introduction of a disturbance: (a) At $=0.00167$ (b) At $=0.00844$ (c) At $=0.0167$. 